\title{
Interactive comment on "On the representation of water reservoir storage and operations in large-scale hydrological models: implications on model parameterization and climate change impact assessments" by Thanh Duc Dang et al.
}

\section{Thanh Duc Dang et al.}

stefano_galelli@sutd.edu.sg

Received and published: 25 September 2019

General Comments:

R: This work contributes to literature on the representation of human interactions in hydrological model by demonstrating the importance of modeling reservoirs. Using the high-profile case of the Lancang / Mekong river basin, they calibrate a large-scale hydrological model (VIC), both with and without reservoirs. They show that while there 
reservoir model compensate the absence of human-operated storage by artificially creating soil storage. Then they go on to investigate the reactions of both models to climate change.

This is an interesting, timely and well-written piece of work that fits well within the scope of HESS. My comments are mainly at how the results are handled and interpreted.

A: We thank the reviewer for the positive feedback.

R: 1) While authors explain very well the consequences of the no-reservoir calibration in terms of structural model behavior, they do not show what that means for water resources appraisals. Indeed, both models are calibrated to have the same behavior downstream of 1 gauge. The crucial difference is that the model including reservoirs can be 1) validated at other (upstream) sites, and 2) validated with post 2005 dams (or the results can be extrapolated with new dams or new operating rules). This point is not really demonstrated.

A: Thanks for this suggestion. We totally agree that we can strengthen our message by implementing one of these two validations. In particular, we believe that the first option is the most feasible, although it presents some minor challenges on which we elaborate below. (Our thoughts regarding the second option are outlined as a response to comment no. 4.)

Discharge data in the Chinese section of the Mekong basin are not available to the international research community. More specifically, only water level data for the flood season (June-October) at Yunjinghong station (located downstream of Jinghong reservoir; see Figure 1 in the manuscript) are available through the Mekong River Commission (http://www.mrcmekong.org/news-and-events/news/mrc-and-chinarenew-pact-on-water-data-provision-and-other-cooperation-initiatives/). Therefore, we opted for retrieving data from three previous studies (He et al. (2009), Wang et al. (2018), and Tang et al. (2019)) that published monthly discharge values at Jiuzhou station (located near Gonguoqiao reservoir; see Figure 1) for the period 1996-2005.

Printer-friendly version

Discussion paper 
When retrieving (digitalizing) the data, we found a maximum discrepancy between the three time series of about $10 \mathrm{~m} 3 / \mathrm{s}$; a negligible value if we consider that the minimum discharge is roughly $400 \mathrm{~m} 3 / \mathrm{s}$.

With this 10-year time series, we can then carry out a thorough validation exercise, whose result is illustrated in Figure 1 (of this document). We can note that the model calibrated without reservoirs largely overestimates the dry season flow and slightly underestimates the wet season flow; a result confirmed by the values of NSE and TRMSE. This result corroborates our finding that during the dry season the model without reservoirs generates more baseflow and runoff than the model with reservoirs (and vice versa during the wet season).

R: 2) Likewise, I disagree that the results from climate models are very different. Yes, there are some small difference, and authors do a good job of explaining them, but these differences are arguably small compared with the uncertainty surrounding downscaled climate projections. In fact, it is remarkable how robust the no-reservoir model results are with climate change.

A: We agree with the reviewer that the uncertainty surrounding the climate change projections is larger than the difference (in discharge) due to the representation of water reservoirs in VIC. This said, we also note that such difference is non-negligible and consistent across both RCPs. We will thus proceed by slightly toning down our conclusions on this result in the revised version of the manuscript.

R: 3) I would advise authors to try and show how both calibrations differ at upstream gauges (where only the with-reservoir calibration would yield sensible results) where there is data, then carry on that comparison with climate change.

A: Yes, we will include an additional validation based on data at an upstream gauge, as explained in our reply to comment no. 1 . We will also include the same comparison under the climate change scenarios. 
R: 4) Alternatively (but this is probably more work), authors can focus on post-2005 years with the addition of new dams and look at the differences between both model at the outlet. And again look at how the two models differ with climate change then.

A: The validation based on discharge data collected in the upstream reaches of the basin is indeed simpler to implement, so we prefer to adopt such option. This said, we understand the potential of this second suggested validation, so we will elaborate on this point in the concluding remarks (please also refer to our answer to the last minor comment).

R: 5) Figure 8 is not very clear and could be replaced with curves comparing average monthly basinwide runoff for the two calibrations (and same for baseflow). This would show explicitly how the no-reservoir calibration compensates for the reservoirs. Alternatively, authors could map the dry / wet season cell-by-cell differences in baseflow / runoff between the two calibrations.

A: Yes, we agree that Figure 8 is indeed not clear. The option of replacing it with curves comparing average monthly basin-wide runoff for the two calibrations is sound. Yet, such figure would not represent explicitly the spatial variability or runoff and baseflow; something that we deem important. We thus prefer to keep the current 'skeleton' of Figure 8, which we will improve with two changes: 1) modify the number of baseflow intervals (as suggested by Reviewer \#1), and 2) use the average annual values of simulated baseflow and runoff (instead of seasonal). A preliminary version of the new figure is given in Figure 2) (of this document).

R: 6) Figures 9 and 10 don't bring much and could be relegated to supplementary material to make place for new figures that can show the consequences of omitting reservoirs, e.g., as suggested in 3) and 4).

Printer-friendly version

A: Yes, we will move both Figure 9 and 10 to the supplement.

Detailed Comments:

Discussion paper 
R: Figure 1.b: the scale, useful in 1.a, is missing.

A: We will add the scale to Figure 1.b.

R: Section 3.1.2: a short description of the version 4.2 of VIC's routing module would be helpful here. Recall that this research is useful for all large-scale hydrological models, not just for VIC experts.

Interactive

A: We will provide a brief description of VIC's routing module. However, we think it's better to include such description in Section 3.1.1, which focuses entirely on VIC (section 3.1.2 is about the modification we introduced). More specifically, we will add the following paragraph: "This module takes as input the gridded surface runoff and baseflow produced by the rainfall-runoff module. For each cell, it then implements a Unit Hydrograph approach to transport the runoff and baseflow to the outlet of that gridunder the assumption that all runoff exits a cell in a single flow direction. Finally, it simulates the channel routing using the linearized Saint-Venant equation."

R: Page 8, lines 2-9: there is a recent publication presenting a database of reservoir storage-area-depth relationships in Yigzaw et al (2018) there https://agupubs.onlinelibrary.wiley.com/doi/10.1029/2017WR022040 could that affect your results in significant ways?

A: Thanks for pointing us to this paper. We noticed that only Manwan reservoir is in this new database, so we believe this cannot impact our model implementation / results. This said, we believe that the adoption of Liebe's method should provide robust results-the method has been tested and adopted by several state-of-the-art studies in large-scale hydrology.

R: Page 9, lines 8-12: how many parallel processors are running? (I. 12) by runtime, do you mean the wall clock time or the total computational time used by all the processors (i.e. wallclock time times \# of processors)? How do we know that 20 seeds are enough? And that the algorithm has converged after 250 function evaluations? Finally, what is

Printer-friendly version

Discussion paper 
the runtime for one run of VIC for that basin?

A: For each (of the 20) seed(s), we used four cores. As for the runtime, we reported the wall-clock time per core. The runtime of VIC for the basin is about 50 mins (on one core). We will clarify these details in the revised version of the manuscript.

There are no specific guidelines in the literature on the number of seeds that should be used (this also depends on the specific MOEA that one adopts). In general, the idea is to use at least 10 seeds, so as to contribute diversity to a given MOEA's search results. Naturally, the number of seeds that one can use depends on the computational requirements of the simulation model as well as the available computational power.

To measure the convergence of the optimization algorithm, we used the hypervolume indicator, which captures both convergence and diversity of a Pareto front (see Reed et al., (2013)). Results indicated that the algorithm reached convergence after $\sim 150$ iterations.

R: Page 14, lines 14-17: I disagree this is a real limitation, since proposing a universal rule system for reservoir operations is not an aim of this paper. Instead, a consequence of your work is that hydrological model calibration with reservoirs and a bespoke release rule means that the model still captures key hydrological processes once the release rules change (because there are new reservoirs / because reservoirs' purposes evolve). In contrast, if reservoirs are not represented to begin with, once the number and / or operations of reservoirs evolves, the model's hydrological parameters have to be recalibrated every time.

A: Thanks for this comment. We indeed said that this is not a limitation (see line 15), but we agree that the sentence was structured in an awkward-and potentially misleading-way. We will proceed by modifying this part of the Conclusions, so as to better emphasize the importance of a correct representation of water reservoirs in large-scale hydrological models. 


\section{References}

He, D., Lu, Y., Li, Z., Li, S. (2009). Watercourse environmental change in Upper Mekong. The Mekong. Academic Press, 335-362.

Reed, P.M., Hadka, D., Herman, J.D., Kasprzyk, J.R., Kollat, J.B. (2013). Evolutionary multiobjective optimization in water resources: The past, present, and future. Advances in Water Resources, 51, 438-456.

Tang, X., Zhang, J., Wang, G., Yang, Q., Yang, Y., Guan, T., Liu, C., Jin, J., Liu, Y., Bao, Z. (2019). Evaluating Suitability of Multiple Precipitation Products for the Lancang River Basin. Chinese Geographical Science, 29(1), 37-57.

Wang, Z., Chen, J., Lai, C., Zhong, R., Chen, X., Yu, H. (2018). Hydrologic assessment of the TMPA 3B42-V7 product in a typical alpine and gorge region: the Lancang River basin, China. Hydrology Research, 49(6), 2002-2015.

Interactive comment on Hydrol. Earth Syst. Sci. Discuss., https://doi.org/10.5194/hess-2019334, 2019. 


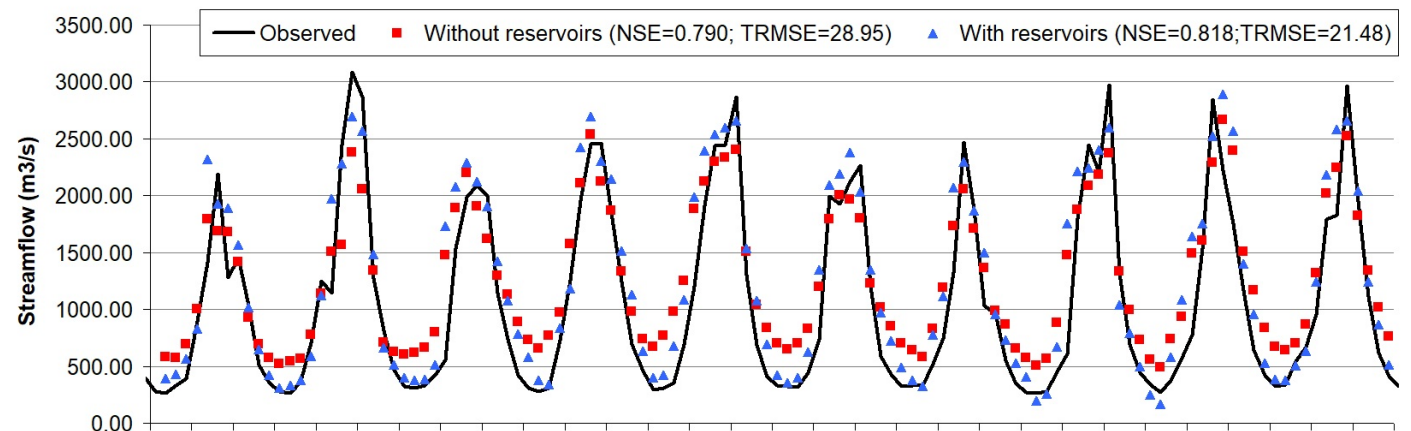

(a)

Fig. 1. Observed versus modelled streamflow time series at Jiuzhou station. 


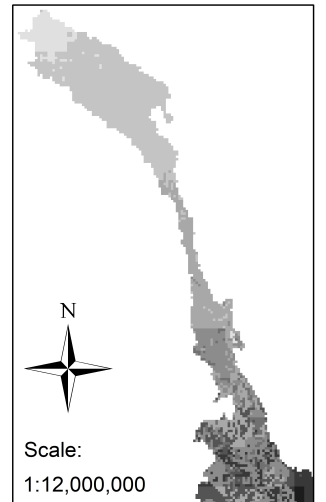

(a) Baseflow - Without reservoirs Legend (unit: $\mathrm{m} 3 / \mathrm{s}$ )

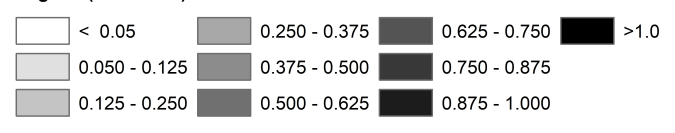

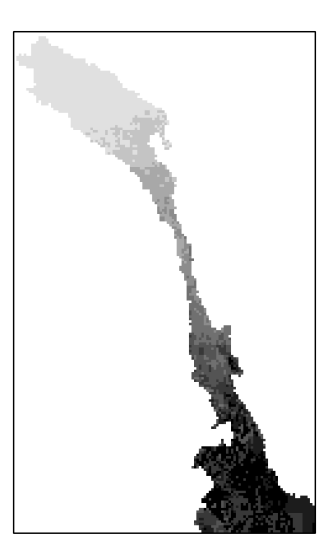

(c) Runoff - Without reservoirs

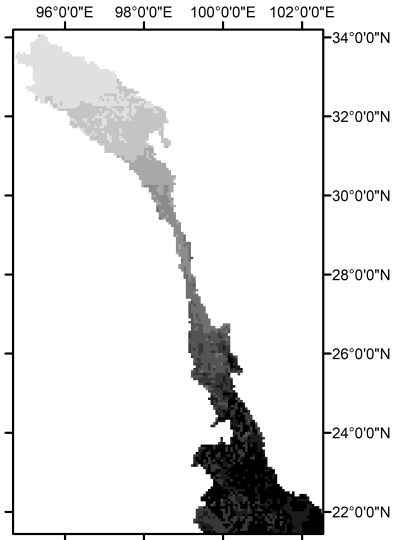

(d) Runoff - With reservoirs

Interactive

comment

\begin{tabular}{|c|c|c|}
\hline$<0.10$ & $0.625-0.750$ & $1.000-1.125$ \\
\hline $0.100-0.500$ & $0.750-0.875$ & $1.125-1.250$ \\
\hline $0.500-0.625$ & $0.875-1.000$ & $1.250-1.375$ \\
\hline
\end{tabular}

Fig. 2. Average annual values of simulated baseflow $(a, b)$ and runoff $(c, d)$ simulated by the selected models (with and without reservoir) during the period 1996-2005. 